\title{
Role of interventional radiology in the management of complications after pancreatic surgery: a pictorial review
}

\author{
Giovanni Mauri • Chiara Mattiuz • Luca Maria Sconfienza • Vittorio Pedicini • \\ Dario Poretti • Fabio Melchiorre • Umberto Rossi • Fabio Romano Lutman • \\ Marco Montorsi
}

Received: 18 September 2014 / Revised: 1 November 2014 / Accepted: 18 November 2014 / Published online: 17 December 2014

(C) The Author(s) 2014. This article is published with open access at Springerlink.com

\begin{abstract}
Pancreatic resections are surgical procedures associated with high incidence of complications, with relevant morbidity and mortality even at high volume centres. A multidisciplinary approach is essential in the management of these events and interventional radiology plays a crucial role in the treatment of patients developing post-surgical complications. This paper offers an overview on the interventional radiolog-
\end{abstract}

\section{G. Mauri $\cdot$ L. M. Sconfienza}

Servizio di Radiologia, IRCCS Policlinico San Donato,

San Donato Milanese, Milano, Italy

L. M. Sconfienza

e-mail: io@lucasconfienza.it

G. Mauri

Dipartimento Aziendale di Oncologia, Struttura Complessa di

Radiologia Oncologica Interventistica, Azienda Ospedaliera

Ospedale di Circolo di Busto Arsizio, Varese, Italy

C. Mattiuz

Facoltà di Medicina e Chirurgia, Scuola di Specializzazione in Radiodiagnostica, Università degli Studi di Milano, Milano, Italy

e-mail: chiara.mattiuz@gmail.com

\section{M. Sconfienza}

Dipartimento di Scienze Biomediche per la Salute, Università degli

Studi di Milano, San Donato Milanese, Milano, Italy

\section{Pedicini $\cdot$ D. Poretti $\cdot$ F. R. Lutman}

Dipartimento di Diagnostica per Immagini,

IRCCS Istituto Clinico Humanitas, Rozzano, Italy

V. Pedicini

e-mail: vittorio.pedicini@humanitas.it

D. Poretti

e-mail: dario.poretti@humanitas.it

F. R. Lutman

e-mail: Romano.lutman@humanitas.it ical procedures that can be performed to treat different type of complications after pancreatic resection. Procedures such as percutaneous drainage of fluid collections, percutaneous transhepatic biliary procedures, arterial embolisation, venous interventions and fistula embolisation are viable treatment options, with fewer complications compared with re-look surgery, shorter hospital stay and faster recovery. A selection

\section{F. Melchiorre}

Dipartimento di Diagnostica per Immagini,

Azienda Ospedaliera San Paolo, Milano, Italy

e-mail: fabio.melchiorre@ao-sanpaolo.it

U. Rossi

Dipartimento di Scienze Diagnostiche,

Azienda Ospedaliera San Carlo Borromeo, Milano, Italy

e-mail: urossi76@hotmail.com

\section{Montorsi}

Dipartimento di Chirurgia, Azienda Ospedaliera Humanitas (Humanitas Research Hospital), Rozzano, Italy e-mail: marco.montorsi@humanitas.it

\footnotetext{
G. Mauri $(\bowtie)$

Servizio di Radiologia, IRCCS Policlinico San Donato, Piazza Malan 2, 20097 San Donato Milanese, Milano, Italy e-mail: vanni.mauri@gmail.com
} 
of cases of complications following pancreatic surgery managed with interventional radiological procedure are presented and discussed.

\section{Teaching Points}

- Interventional radiology is crucial to treat complications after pancreatic surgery

- Percutaneous drainage of collections can be performed under ultrasound or computed tomography guidance

- Percutaneous biliary procedures can be used to treat biliary complications

- Venous procedures can be performed effectively through transhepatic or transjugular access

- Fistulas can be treated effectively by percutaneous embolisation

Keywords Interventional radiology •

Duodenopancreasectomy · Complication · Embolisation .

Biliary drainage

\section{Introduction}

Pancreatic resection is a surgical procedure associated with significant morbidity and mortality even at specialised highvolume centres [1-3]. Although in recent years refinements in surgical technique and perioperative management have led to a reduction in perioperative mortality, the incidence of postoperative complications (including intra-abdominal abscesses and leakages [4-9], biliary complications [10-14] and vascular complications [15-18]) still remains high. Moreover, in case of complications, surgical re-operation is associated with a high mortality rate [1-3]. In a large series of 650 patients, Yeo et al. [2] reported an overall mortality of $1.4 \%$ and a morbidity of $41 \%$, with a mean length of hospital stay of 13 days. They reported reoperation in $26 / 650$ patients ( $4 \%$ ) and identified the absence of reoperation as an independent predictor of prolonged survival [2].

Interventional radiology (IR) provides a minimally invasive alternative for managing post-surgical complications $[19,20]$. Several different IR procedures, such as percutaneous drainage, aspiration of abscesses or fluid collections $[4,5]$, percutaneous transhepatic biliary drainage [6], and arterial embolisation [21-23] have been introduced in clinical practice to treat post-surgical complications. IR procedures are an alternative approach to manage post-surgical complications less invasive than surgical re-intervention, and may lead to a reduction in hospital stay and re-operation rate $[10,11]$.

In the present pictorial review, we offer an overview on the IR procedures that can be performed to treat different types of complications after pancreatic resection.

\section{Percutaneous drainage}

Intra-abdominal collections and abscesses represent the most common complication following pancreatic surgery [1-3]. Once an intra-abdominal collection is identified, in most cases it is possible to place a percutaneous drainage under image guidance. When the collection is well visible using ultrasound (US), US-guided percutaneous drainage placement is generally the preferred choice, as US is widely available, easy to handle and allows for real-time monitoring of the drainage placement, being also free from ionising radiation [24]. When the collection is located deep in the abdomen and is not well seen at US, computed tomography (CT) generally offers good anatomical definition to guide the safe placement of a percutaneous drainage. Drainage placement can be performed using the trocar or Seldinger technique. In the trocar technique, the drainage catheter containing a trocar needle is inserted directly into the collection. The trocar technique provides a fast deployment of the drainage that can be extremely helpful in critically ill or agitated patients. The Seldinger technique implies multiple steps: the collection is punctured with a small calibre needle, different calibre guidewires are inserted and the drainage is then advanced up to the collection over the guidewire. The Seldinger technique is particularly helpful when there is a small window to reach the collection, as typically happens when a retroperitoneal collection has to be drained through an anterior approach. The main disadvantage of this technique is that it may require considerably longer time than the trocar technique. The correct location of the needle or drainage can be confirmed by aspiration of a small amount of material. Furthermore, a small amount of iodinated contrast may be injected through the catheter to identify the presence of an underlying fistula.

In a series of 373 subjects who underwent pancreaticoduodenectomy reported by Zink et al. [5], percutaneous drainage was required in $22.2 \%$ of patients after surgery. They report an immediate technical and overall success rate of 97.6 and $79.6 \%$, respectively.

A case of a patient with fluid collection after pancreaticoduodenectomy successfully managed with percutaneous CT guided drainage is shown in Fig. 1.

\section{Percutaneous transhepatic biliary procedures}

In patients treated by pancreaticoduodenectomy with Rouxen-Y bilio-enteric reconstruction, endoscopical access to the biliary tree is not feasible. Thus, in this kind of patient, a percutaneous transhepatic route is the only approach to the biliary system in case of biliary complications. The access to the biliary system is obtained by puncturing a peripheral bile duct under US and/or fluoroscopic guidance. 
Fig. 1 Use of a percutaneous drainage to treat a postpancreaticoduodenectomy fluid collection. a CT scan shows postpancreaticoduodenectomy retroperitoneal fluid collection (asterisks). b The fluid collection (asterisks) is punctured with a small needle under CT guidance. c A percutaneous drainage (arrow) is inserted into the collection (asterisks) using the Seldinger technique. $\mathbf{d}$ At the end of the treatment complete resolution of the collection is obtained
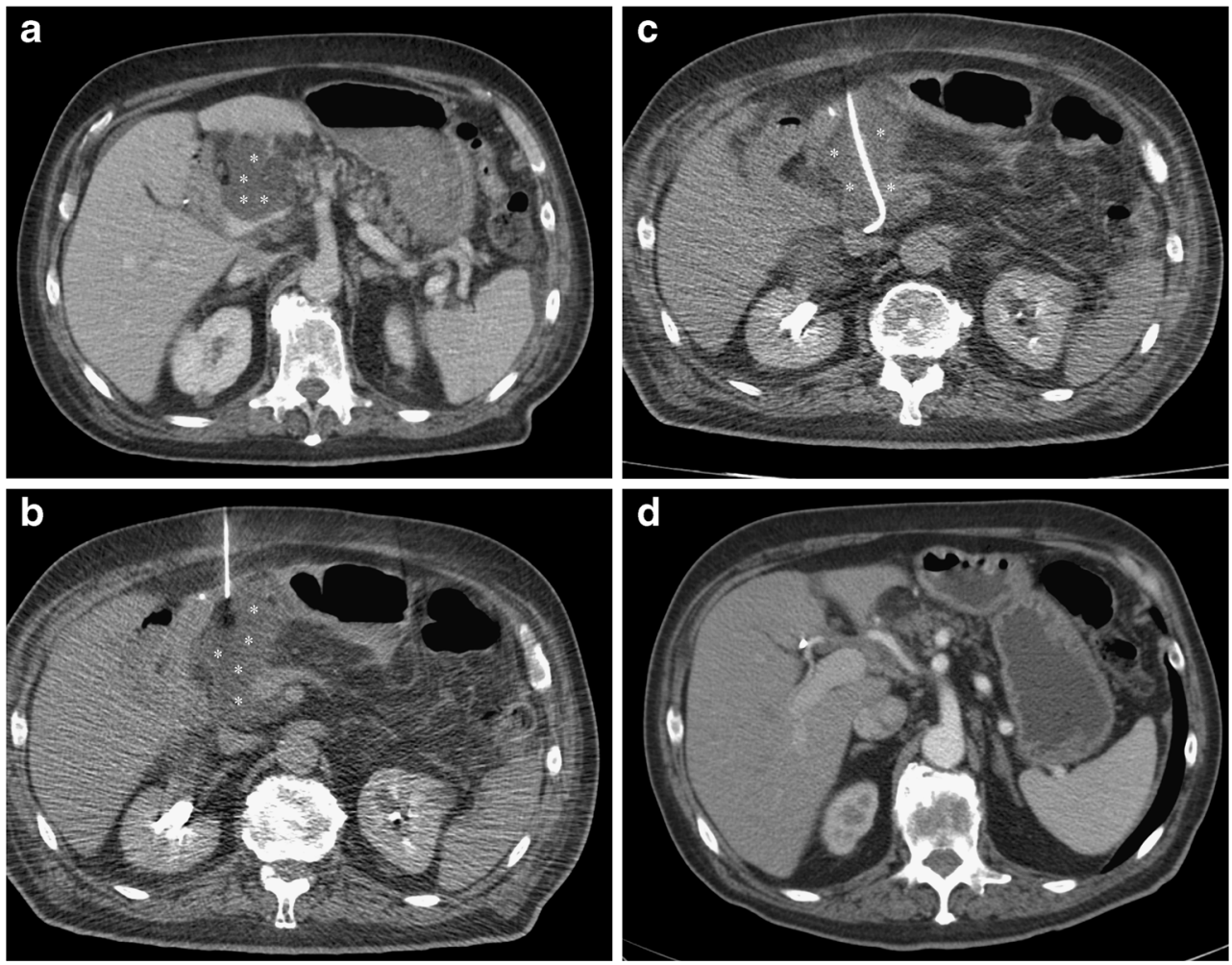

Percutaneous biliary procedures can be particularly helpful in postoperative biliary leaks. They have been reported in about 3-4\% of cases after pancreatic surgery [1-3]. These complications are often associated with others, in particular with pancreatic fistula and fluid collections. In patients who develop such complication after surgery, percutaneous transhepatic biliary drainage - by allowing for a bile diversion from the site of the fistula - has been proven to be feasible and effective in the majority of cases $[6,20,21]$. The positioning of an occlusion balloon to obstruct the biliary duct above the

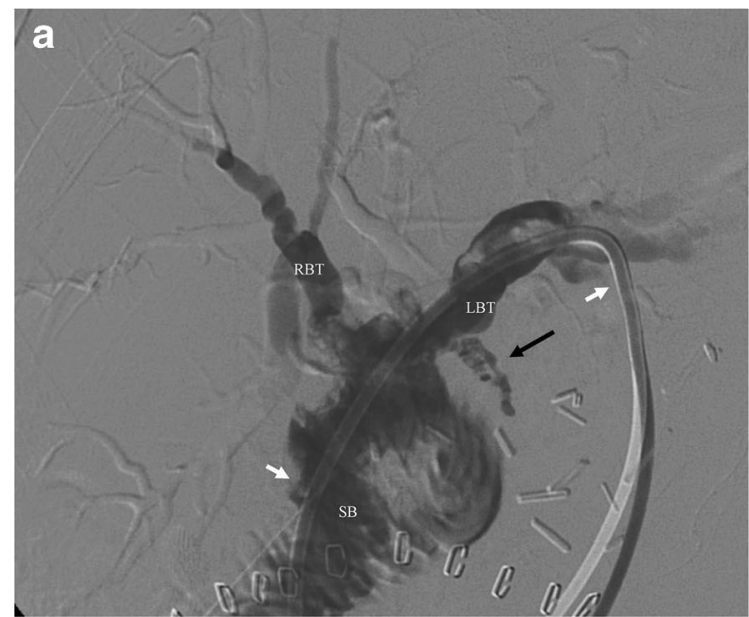

Fig. 2 Use of a percutaneous transhepatic biliary drainage to treat a postsurgical biliary leak. a Percutaneous colangiography demonstrates a biliary leak (arrow) and a biliary drainage (white arrows) is inserted via segment site of the fistula, by allowing for complete external drainage of the bile, may represent a valuable option to treat postsurgical biliary leaks [7, 10]. A different and more recent technique is to use a covered stent that can close the bile leak and subsequently be retrieved percutaneously. Gwon et al. [25] used this approach successfully in 11 patients with postoperative bile leak, with no recurrence in the 1-year follow-up. A case of a patient with post-surgical biliary leak successfully treated with percutaneous transhepatic biliary drainage is shown in Fig. 2.

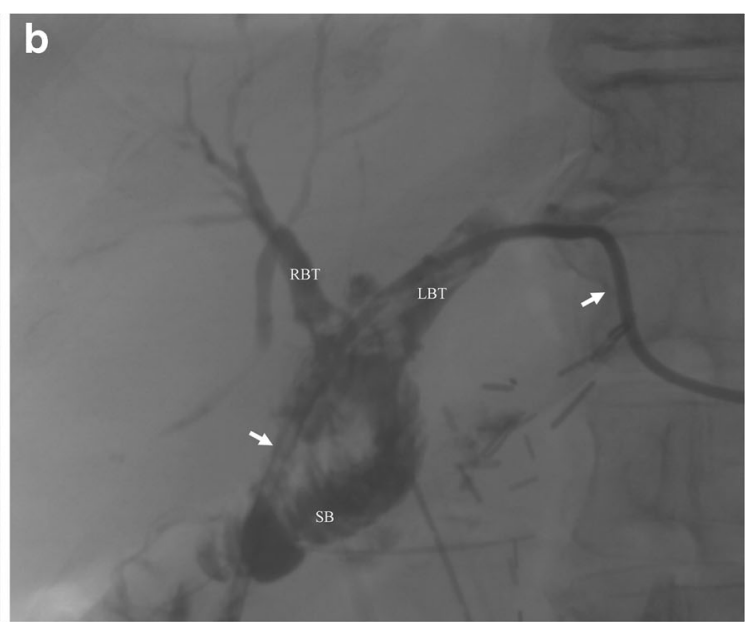

III left lobe approach; $R B T$ right biliary tree, $L B T$ left biliary tree, $S B$ small bowel. b Final result with complete healing of the leak and absence of contrast leakage; $R B T$ right biliary tree, $L B T$ left biliary tree, $S B$ bowel 
After bilio-pancreatic surgery, stricture of a biliary duct may represent a serious complication, [26]. Surgical reintervention is associated with morbidity and mortality rates as high as 28 and $2.6 \%$ respectively [26]. Percutaneous treatments represent an effective alternative to surgery in the treatment of such complication [27-29]. To achieve resolution of the stenosis, subsequent larger biliary drainage catheter may be inserted and left in place and balloon dilation can be performed. However, recurrence of stenosis may occur in up to $29-58 \%$ of cases [27-29], and multiple treatment sessions may be required. Stents are rarely used in the treatment of benign strictures, as they have to be removed after a period because the tube itself may stimulate inflammatory reaction, fibrosis and stone formation. A novel option may be represented by the use of biodegradable biliary stents, which may improve the long-term patency rate, without requiring a subsequent procedure for removal [13].

A case of a patient with a post-surgical biliary stricture successfully treated with balloon dilation and placement of a biodegradable biliary stent is shown in Fig. 3.

\section{Arterial embolisation}

Postoperative intra-abdominal arterial haemorrhage is still one of the most serious complications, with a reported incidence between 1.5 and $15 \%$, and a reported mortality rate of 20 $50 \%$ [15-17]. Haemorrhage is defined as 'early' when occurring within $24 \mathrm{~h}$ after surgery, while 'late' haemorrhages occur after $24 \mathrm{~h} \mathrm{[30].} \mathrm{Early} \mathrm{haemorrhage} \mathrm{requires} \mathrm{immediate}$

Fig. 3 Percutaneous treatment of a benign stricture of the common bile duct by a bioabsorbable biliary stent. a Percutaneous transhepatic cholangiography demonstrates remarkably dilated bile ducts (asterisks). The obstruction can be clearly spotted (white arrows). b Bilioplasty procedure. The balloon (black arrows) is fully inflated to dilate the stricture. Radiopaque contrast agent can be detected in the bowel $(B)$; asterisks dilated bile ducts. c A bioabsorbable stent is positioned to keep the stricture open. The stent is radiolucent, but two platinum, radiopaque markers can be seen (black arrowheads); asterisks dilated bile ducts, $B$ bowel. d The stent is fully expanded and radiopaque bile can be seen flowing through the patent common bile duct (white arrowheads); black arrows radiopaque stent markers; $B$ bowel. e US follow-up at 3 months demonstrating good visibility of the stent (arrowheads) that was correctly expanded, and no dilation of the intrahepatic biliary ducts; $L$ liver
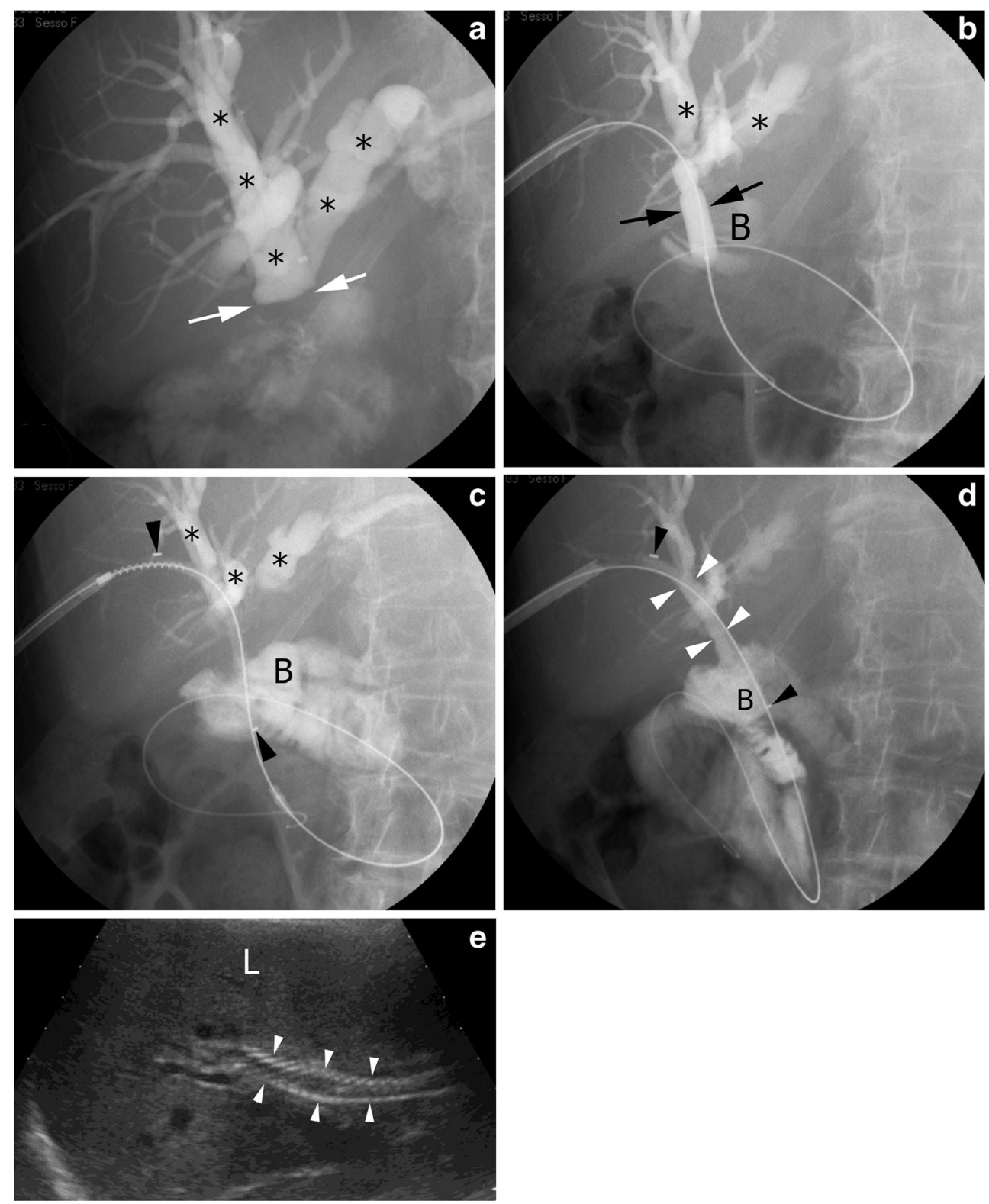
laparotomy, as it is generally caused by a technical failure or underlying coagulopathy, while the most appropriate management of delayed haemorrhage still remains controversial. In haemodynamically stable patients, CT is crucial to address the suspicion of a delayed haemorrhage and is extremely useful prior to angiography as it may avoid it or guide it. In unstable patients, direct visceral angiography has been suggested as the best method to elucidate the site of bleeding, with the advantage of sparing time for a subsequent immediate intra-arterial treatment.

Transcatheter arterial embolisation has been reported to be safe and effective, with a reported success rate of 50-100 \%, gaining acceptance for the treatment of intra-abdominal bleeding [15-17]. Once the site of bleeding has been identified, several different techniques may be used to stop bleeding and achieve haemostasis. In case of terminal vascularisation, a proximal embolisation of the bleeding vessel may be enough to achieve the haemostasis, while in the presence of collaterals, it is crucial to embolise both the inflow and outflow vessels in order to avoid re-bleeding ("isolation technique") [22]. Different materials are nowadays available to obtain vascular occlusion, and have to be chosen according to the desired type of vascular occlusion (transient or permanent). Transient embolisation, generally required due to a traumatic haemorrhage, is reached by resorbable materials, which allows for restoring the blood flow in a variable window of time (inter alia: autologous blood clot, gelatine or fibrin sponge). The main advantage of such materials is to avoid definitive occlusion of the treated vessel. However, a non-negligible risk of re-bleeding has to be considered once the material has been absorbed. Permanent embolisation is reached using nonresorbable materials (polyvynilic alcohol, bucrilate, metallic coils or detachable balloons) that induce a permanent vessel occlusion. At the present, an ideal material does not exist, thus the choice of the most appropriate embolic material and technique for embolisation, crucial to minimise failure and complications, requires the presence of a IR team with high experience and the availability of several different materials.

Stent grafting of the artery at the site of bleeding has been proposed as an alternative or in addition to embolisation, with the advantage of maintaining the patency of the end organ, thus minimising the risk of ischaemia deriving from embolisation [21]. A recent meta-analyisis comparing laparotomy and transarterial embolisation for the management of delayed
Fig. 4 Arterial embolisation of a post-surgical haemorrhage. a CT scan showing contrast extravasation (arrow) from the celiac trunk at the level of the bifurcation between hepatic and splenic artery; $V$ inferior vena cava, $A$ abdominal aorta. b Angiographic appearance of the active bleeding with contrast extravasation (arrows); asterisk celiac trunk, black arrowhead splenic artery, white arrowhead hepatic artery. c Angiography after embolisation with coils (arrows) demonstrating absence of contrast extravasation at the level of previous bleeding. d CT scan at 2 years,

demonstrating absence of contrast extravasation at the level of previous bleeding and preserved patency of proper hepatic artery (white arrow), even in the presence of part of a coil in its lumen (arrowhead); black arrow clustered metallic coils in the focus of arterial lesion properly embolised
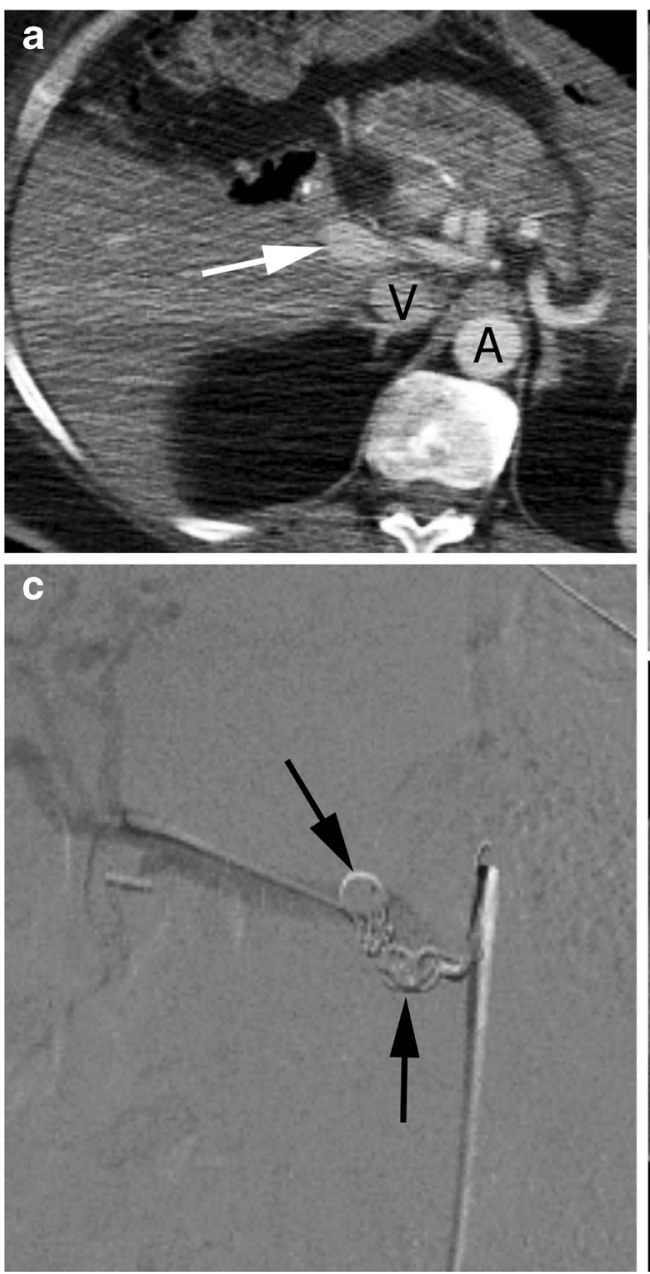
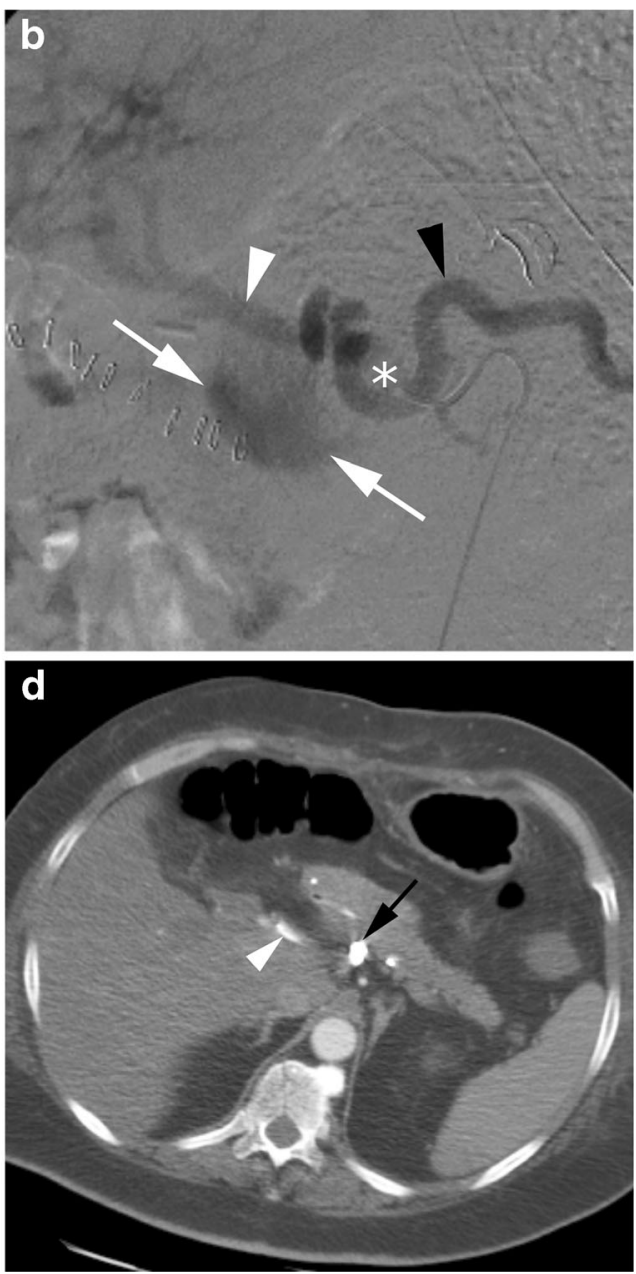
postoperative haemorrhage found a reduction in mortality (43\% vs $20 \%$ ) and morbidity (77\% vs $35 \%$ ) in the IR group, even if not reaching the statistical significance. Authors conclude that the appropriate treatment pathway for late haemorrhage ultimately will be decided by the clinical status of the patient and the institution preference [16].
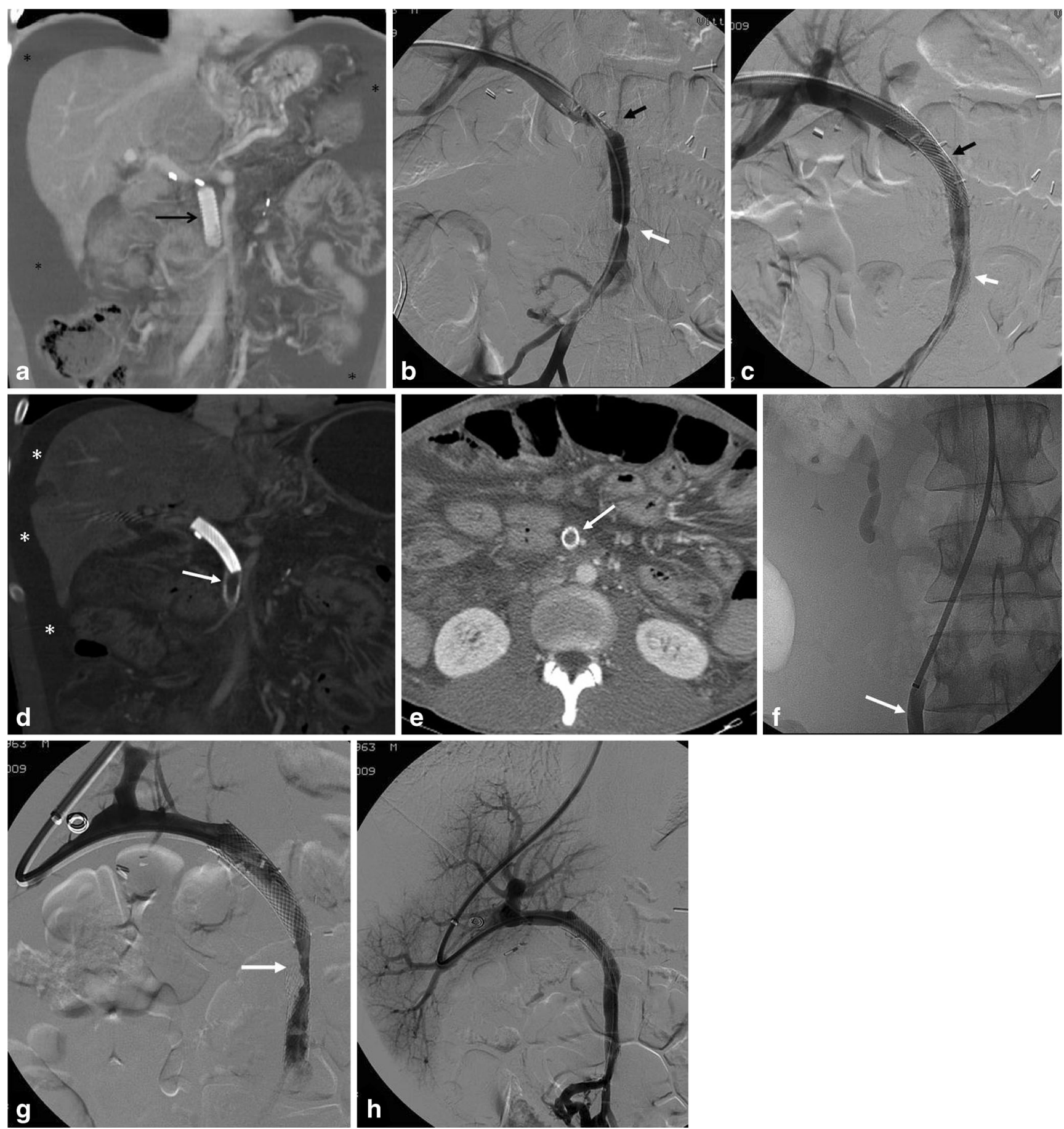

Fig. 5 Treatment of a patient with postoperative mesenteric vein stenosis and thrombosis by percutaneous transhepatic stenting, thromboaspiration, and thrombolysis through a transjugular approach (the case has been partially previously reported [18]). a Maximum intensity projection reconstruction of abdominal MDCT shows surgical vascular graft (black arrow) at the level of the superior mesenteric vein and ascitic fluid (asterisks) b Percutaneous transhepatic angiography demonstrates stenosis at the level of proximal (black arrow) and distal (white arrow) anastomoses

of the PTFE graft. c Angiography after stent placement (black arrow proximal stent, white arrow distal stent) demonstrating resolution of the stenosis. $\mathbf{d}$ MDCT MIP reconstruction and $\mathbf{e}$ axial image showing intra-stent thrombosis (white arrow) and ascitic fluid (asterisks). f Contrast injection in SMV (arrow) demonstrates complete vessel occlusion. g Angiography after aspiration thrombectomy and angioplasty shows residual thrombus (arrow). h Complete resolution and calibre restoration after direct thrombolysis with rt-PA 
A case of transcatheter arterial embolisation for a postsurgical haemorrhage is shown in Fig. 4.

\section{Venous interventions}

In some cases, post-surgical complications may involve the portal and mesenteric veins, which may develop stenosis or thrombosis after surgical treatment. Moreover, with the recent advancement of vascular reconstruction with polytetrafluoroethylene graft, this occurrence may happen more frequently in the future. Percutaneous endovascular treatment, such as transjugular portosystemic shunt, direct and indirect thrombolysis, stenting and mechanical thrombectomy, described mainly in liver-transplant patients, may represent a valuable option in the management of these conditions after pancreatic surgery $[2$, $5,18]$. The access to the portal system may be achieved through a transhepatic route, with the direct image guided puncture of a peripheral portal branch, or through a transjugular approach, by puncturing the portal system from the hepatic veins. Transhepatic access is generally easier than transjugular access and represents the first choice strategy in patients with normal coagulation parameters. In patients with thrombosis of the portal or mesenteric vein, in which anticoagulation treatment has been undertaken, the best choice is represented by the transjugular approach, in order to minimise the risk of peritoneal haemorrhage correlated with direct liver puncture [18]. Once the access to the portal system has been achieved, it is possible to perform balloon dilation and stenting of post-surgical strictures using the several different commercially available devices. In case of thrombosis, direct trombo-aspiration through a catheter or infusion of trombolitic agents has been reported as a feasible and effective treatment [18].

A case of post-surgical mesenteric vein stenosis and thrombosis treated percutaneously is shown in Fig. 5 .

\section{Fistula embolisation}

Fistulas are the most common complication following pancreatic surgery after presence of fluid collections [1-3]. The most frequent type is pancreatic fistula, followed by enteric and biliary fistulas [1-3]. This kind of complication is frequently associated with others, in particular the presence of an intraabdominal fluid collection. Recent reports highlighted how this kind of complication can be successfully managed
Fig. 6 Successful percutaneous embolisation with cyanoacrilic glue of a postoperative fistula. a, b Contrast injection through a previously placed percutaneous drainage (white arrowhead) at the level of a fluid collection (asterisk) demonstrating a fistula (black arrow) with a communication (small black arrowhead) with the biliary system and bowel $(B)$; large black arrowhead percutaneous transhepatic biliary drainage. $\mathbf{c} \mathrm{A}$ guidewire (small white arrowhead) is advanced through the percutaneous drainage (large white arrowhead) into the fistula (black arrow); asterisk fluid collection, black arrowhead percutaneous transhepatic biliary drainage, $B$ bowel. d A microcatheter (white arrowhead) is advanced over the wire at the level of the fistula (black arrow), and glue is injected (white arrow); asterisk fluid collection, black arrowhead percutaneous transhepatic biliary drainage, $B$ bowel
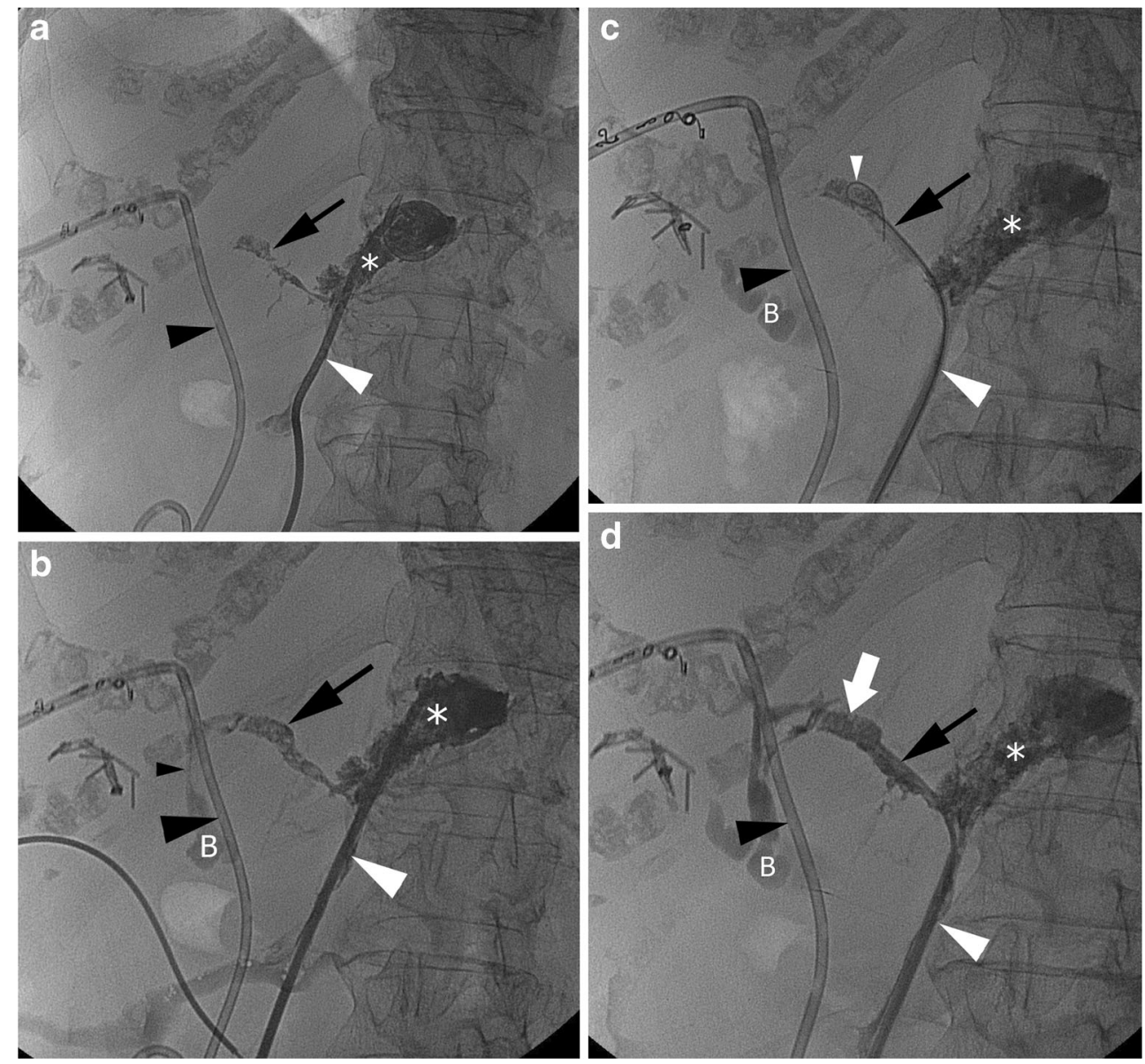
without surgery in over $90 \%$ of patients [9]. In the nonoperative management of such complications, IR plays a crucial role.

In presence of a fluid collection, a percutaneously placed drainage may be enough to treat the collection, and in several cases to allow for the spontaneous closure of the fistula [4, 5]. If the main component of a fistula is biliary material, the transhepatic insertion of a biliary drainage, by diverting the bile from the site of the fistula, may be enough to determine the fistula closure. In cases with persisting biliary leak, the placement of an occlusion balloon above the fistula, by interrupting completely the bile flow towards the site of the fistula, has been reported as an effective tool [7-10].

Some authors reported the direct embolisation of the site of the fistula as a feasible and effective procedure. Reaching perfectly the site of the fistula is crucial to perform a correct procedure. This can be done through a previously placed surgical drainage, through an imageguided percutaneously placed drainage, or even through a transhepatic approach. Once the site of the fistula has been reached, several different materials can be used to perform embolisation, including ethanol, particles or different kind of glues [31-33]. Inter alia, cyanoacrilic glues seem to represent optimal materials for fistula percutaneous treatment, due to their high adhesive and haemostatic properties and fast polymerisation [31].

A case of embolisation of a fistula with cyanoacrilic glue is shown in Fig. 6.

\section{Conclusions}

IR plays an increasing, crucial role in the multidisciplinary management of complications after pancreatic surgery, providing a minimally invasive therapy also in critical patients, reducing recovery times and avoiding re-operation morbidity. IR procedures such as percutaneous drainage of fluid collections, percutaneous transhepatic biliary procedures, arterial embolisation, venous interventions and fistula embolisation are viable treatment options and have been reported as feasible, safe and effective techniques with fewer complications compared with re-look surgery, with a shorter hospital stay and faster recovery in the management of complications after pancreatic surgery.

Conflict of interest Giovanni Mauri declares sponsored research and consulting from Esaote S.p.A, Italy. All other authors declare no conflicts of interest.

Open Access This article is distributed under the terms of the Creative Commons Attribution License which permits any use, distribution, and reproduction in any medium, provided the original author(s) and the source are credited.

\section{References}

1. Buchler MW, Wagner M, Schmied BM, Uhl W, Friess H, Z'Graggen $\mathrm{K}$ (2003) Changes in morbidity after pancreatic resection: toward the end of completion pancreatectomy. Arch Surg 138(12):1310-1314

2. Yeo CJ, Cameron JL, Sohn TA et al (1997) Six hundred fifty consecutive pancreaticoduodenectomies in the 1990s: pathology, complications, and outcomes. Ann Surg 226(3):248-257

3. Sohn TA, Yeo CJ, Cameron JL et al (2000) Resected adenocarcinoma of the pancreas-616 patients: results, outcomes, and prognostic indicators. J Gastrointest Surg 4(6):567-579

4. Gervais DA, Fernandez-del Castillo C, O'Neill MJ, Hahn PF, Mueller PR (2001) Complications after pancreatoduodenectomy: imaging and imaging-guided interventional procedures. Radiographics 21(3):673-690

5. Zink SI, Soloff EV, White RR et al (2009) Pancreaticoduodenectomy: frequency and outcome of post-operative imaging-guided percutaneous drainage. Abdom Imaging 34(6):767-771

6. Cozzi G, Severini A, Civelli E et al (2006) Percutaneous transhepatic biliary drainage in the management of postsurgical biliary leaks in patients with nondilated intrahepatic bile ducts. Cardiovasc Intervent Radiol 29(3):380-388

7. Pedicini V, Poretti D, Mauri G et al (2010) Management of post-surgical biliary leakage with percutaneous transhepatic biliary drainage (PTBD) and occlusion balloon (OB) in patients without dilatation of the biliary tree: preliminary results. Eur Radiol 20(5):1061-1068

8. Winter JM, Cameron JL, Yeo CJ, Lillemoe KD, Campbell KA, Schulick RD (2008) Duodenojejunostomy leaks after pancreaticoduodenectomy. J Gastrointest Surg 12(2):263-269

9. Shrikhande SV, D’Souza MA (2008) Pancreatic fistula after pancreatectomy: evolving definitions, preventive strategies and modern management. World J Gastroenterol 14(38):5789-5796

10. Cozzaglio L, Cimino M, Mauri G et al (2011) Percutaneous transhepatic biliary drainage and occlusion balloon in the management of duodenal stump fistula. J Gastrointest Surg $15: 1977-1981$

11. Kim JH, Ko GY, Sung KB et al (2008) Bile leak following living donor liver transplantation: clinical efficacy of percutaneous transhepatic treatment. Liver Transpl 14:1142-1149

12. Mauri G, Sconfienza LM (2014) Few comments on "Defining treatment and outcomes of hepaticojejunostomy failure following pancreaticoduodenectomy". J Gastrointest Surg 18(4):880-881

13. Mauri G, Michelozzi C, Poretti D et al (2013) Bioabsorbable biliary stent implantation in the treatment of benign bilioplastic-refractory biliary strictures: preliminary experience. Eur Radiol 23(12):3304-3310

14. Mauri G, Sconfienza LM (2013) A few thoughts on "Interventional radiology in the management of benign biliary stenoses, biliary leaks and fistulas: a pictorial review" Insights. Imaging 4(2):253

15. Blanc T, Cortes A, Goere D et al (2007) Hemorrhage after pancreaticoduodenectomy: when is surgery still indicated? Am J Surg 194(1):3-9

16. Limongelli P, Khorsandi SE, Pai M et al (2008) Management of delayed postoperative hemorrhage after pancreaticoduodenectomy: a meta-analysis. Arch Surg 143(10):1001-1007

17. Yekebas EF, Wolfram L, Cataldegirmen G et al (2007) Postpancreatectomy hemorrhage: diagnosis and treatment: an analysis in 1669 consecutive pancreatic resections. Ann Surg 246(2):269-280

18. Mauri G, Monti L, Pedicini V (2011) Interventional management of in-stent thrombosis after superior mesenteric vein stenting. EJVES Extra 22(3):27-29

19. Baker TA, Aaron JM, Borge M, Pierce K, Shoup M, Aranha GV (2008) Role of interventional radiology in the management of complications after pancreaticoduodenectomy. Am J Surg 195(3):386390 
20. Sohn TA, Yeo CJ, Cameron JL et al (2003) Pancreaticoduodenectomy: role of interventional radiologists in managing patients and complications. J Gastrointest Surg 7(2):209-219

21. Lee HG, Heo JS, Choi SH, Choi DW (2010) Management of bleeding from pseudoaneurysms following pancreaticoduodenectomy. World J Gastroenterol 16(10):1239-1244

22. Rossi UG, Seitun S, Ferro C (2013) Endovascular embolization of a third jejunal artery aneurysm: isolation techinique using the Amplatzer vascular plug 4. Catheter Cardiovasc Interv 81(6):1049-1052

23. Miura F, Asano T, Amano H et al (2009) Management of postoperative arterial haemorrhage after pancreato-biliary surgery according to the site of bleeding: re-laparotomy or interventional radiology. $\mathrm{J}$ Hepatobiliary Pancreat Surg 16(1):56-63

24. Sconfienza LM, Mauri G, Grossi F et al (2013) Pleural and peripheral lung lesions: comparison of US- and CT-guided biopsy. Radiol 266(3):930-935

25. Gwon DI, Ko GY, Sung KB, Kim JH, Yoon HK (2011) Percutaneous transhepatic treatment of postoperative bile leaks: prospective evaluation of retrievable covered stent. J Vasc Interv Radiol 22(1):75-83

26. Shimada H, Endo I, Shimada K, Matsuyama R, Kobayashi N, Kubota K (2012) The current diagnosis and treatment of benign biliary stricture. Surg Today 42:1143-1153
27. Mueller PR, vanSonnenberg E, Ferrucci JT Jr, Weyman PJ, Butch RJ, Malt RA et al (1986) Biliary stricture dilatation: multicenter review of clinical management in 73 patients. Radiol 160(1):17-22

28. Born P, Rosch T, Bruhl K et al (1999) Long-term results of endoscopic and percutaneous transhepatic treatment of benign biliary strictures. Endosc 31(9):725-731

29. Krokidis M, Orgera G, Rossi M, Matteoli M, Hatzidakis A (2013) Interventional radiology in the management of benign biliary stenoses, biliary leaks and fistulas: a pictorial review. Insights Imaging 4:77-84

30. Wente MN, Veit JA, Bassi C et al (2007) Postpancreatectomy haemorrhage (PPH): an International Study Group of Pancretic Surgery (ISGPS) definition. Surg 142(1):20-25

31. Mauri G, Sconfienza LM, Fiore B et al (2013) Post-surgical enteric fistula treatment with image-guided percutaneous injection of cyanoacrylic glue. Clin Rad 68(1):59-63

32. Sconfienza LM, Mauri G (2014) Interventional options to treat postoperative duodenal stump leaks. Cardiovasc Intervent Radiol. doi:10. 1007/s00270-014-0937-7

33. Mtsumoto T, Iwaki K, Hagino Y et al (2002) Ethanol injection therapy of an isolated bile duct associated with a biliary-cutaneous fistula. J Gastroenterol Hepatol 17(7):807-810 